\title{
Increasing prevalence of anticholinergic medication use in older people in England over 20 years: cognitive function and ageing study I and II
}

Carlota M. Grossi ${ }^{1 \dagger}$, Kathryn Richardson ${ }^{1 \dagger}$, George M. Savva², Chris Fox ${ }^{1}$, Antony Arthur ${ }^{1}$, Yoon K. Loke ${ }^{1}$, Nicholas Steel ${ }^{1}$, Carol Brayne ${ }^{3}$, Fiona E. Matthews ${ }^{4}$, Louise Robinson ${ }^{4}$, Phyo K. Myint ${ }^{5}$ and lan D. Maidment ${ }^{6^{*}}$ (D)

\begin{abstract}
Background: Anticholinergic medication use is linked with increased cognitive decline, dementia, falls and mortality, and their use should be limited in older people. Here we estimate the prevalence of anticholinergic use in England's older population in 1991 and 2011, and describe changes in use by participant's age, sex, cognition and disability.

Methods: We compared data from participants aged $65+$ years from the Cognitive Function and Ageing Studies (CFAS I and II), collected during 1990-1993 ( $N=7635)$ and 2008-2011 ( $N=7762)$. We estimated the prevalence of potent anticholinergic use (Anticholinergic Cognitive Burden [ACB] score $=3$ ) and average anticholinergic burden (sum of ACB scores), using inverse probability weights standardised to the 2011 UK population. These were stratified by age, sex, Mini-Mental State Examination score, and activities of daily living (ADL) or instrumental ADL (IADL) disability.

Results: Prevalence of potent anticholinergic use increased from 5.7\% (95\% Confidence Interval [CI] 5.2-6.3\%) of the older population in 1990-93 to 9.9\% (9.3-10.7\%) in 2008-11, adjusted odds ratio of 1.90 (95\% Cl 1.67-2.16). People with clinically significant cognitive impairment (MMSE [Mini Mental State Examination] 21 or less) were the heaviest users of potent anticholinergics in CFAS II (16.5\% [95\% Cl 12.0-22.3\%]). Large increases in the prevalence of the use medication with 'any' anticholinergic activity were seen in older people with clinically significant cognitive impairment (53.3\% in CFAS I to $71.5 \%$ in CFAS II).
\end{abstract}

Conclusions: Use of potent anticholinergic medications nearly doubled in England's older population over 20 years with some of the greatest increases amongst those particularly vulnerable to anticholinergic side-effects.

Keywords: Cognitive impairment, Anticholinergic burden

\footnotetext{
* Correspondence: i.maidment@aston.ac.uk

${ }^{\dagger}$ Carlota M Grossi and Kathryn Richardson are joint first authors.

${ }^{6}$ Aston University, Birmingham B4 7ET, UK

Full list of author information is available at the end of the article
}

(c) The Author(s). 2020 Open Access This article is licensed under a Creative Commons Attribution 4.0 International License, which permits use, sharing, adaptation, distribution and reproduction in any medium or format, as long as you give appropriate credit to the original author(s) and the source, provide a link to the Creative Commons licence, and indicate if changes were made. The images or other third party material in this article are included in the article's Creative Commons licence, unless indicated otherwise in a credit line to the material. If material is not included in the article's Creative Commons licence and your intended use is not permitted by statutory regulation or exceeds the permitted use, you will need to obtain permission directly from the copyright holder. To view a copy of this licence, visit http://creativecommons.org/licenses/by/4.0/ The Creative Commons Public Domain Dedication waiver (http://creativecommons.org/publicdomain/zero/1.0/) applies to the data made available in this article, unless otherwise stated in a credit line to the data. 


\section{Background}

Globally, the population is ageing; in the UK, the proportion of people aged 65 years or over is projected to increase from $18 \%$ in 2017 to $21 \%$ by 2027 [1]. Multi-morbidity increases with ageing. This increase has been accompanied by a dramatic rise in polypharmacy with the proportion of older people taking five or more medication rising four-fold from 12 to $49 \%$ over 20 years [2]. The concerns about polypharmacy include interactions, burden on patients, side effects, and cost. Many older people frequently receive medicines with anticholinergic properties for diverse conditions, such as depression, bladder problems, Parkinson's disease and chronic obstructive pulmonary disease [3-6].

Anticholinergic activity can cause cognitive decline, falls, constipation and daytime drowsiness in older people [7, $8]$, and worsen cognition and activities of daily living in people living with schizophrenia [9]. Greater cumulative use of anticholinergics has been associated with an increased risk of dementia [10-12], and mortality [7]. Given these possible associations with long term outcomes as well as the known immediate adverse anticholinergic effects, it is widely accepted that these medicines should be avoided in older people where possible [13]. Nevertheless, anticholinergics remain commonly prescribed.

Estimates of prevalence of anticholinergic use vary depending on the population, year and definition of anticholinergic medications. Previous estimates of the prevalence of any anticholinergic use in older adults have varied from 37 to $63 \%[8,14]$, and of 'potent' anticholinergic use from 4 to $10 \%[8,14,15]$. However, less is known about the impact of anticholinergic effects among groups most vulnerable to their side-effects such as older people with clinically significant cognitive impairment including those living with dementia and the very old, because these groups are commonly excluded from clinical trials $[16,17]$.

Medications with anticholinergic activity are most commonly available only by prescription, but are also obtainable over-the-counter. Hence pharmacy dispensing or prescription databases may underestimate the true prevalence of anticholinergic medication use in the population. Prospective longitudinal studies, which aim to ascertain participants' over the counter (OTC) and prescription medication use, may offer the best opportunities to understand changing patterns in their use. These prospective longitudinal studies also allow the disaggregation of older populations by health status and so allow the medication use patterns to be described by physical and cognitive frailty.

The overall aim of the study was to estimate the prevalence of anticholinergic use in England's older population in 1991 and 2011, and describe changes in use by participant's age, sex, cognition and disability.

\section{Methods}

This study compared the prevalence of anticholinergic medication use in the older population of England using baseline data from two prospective longitudinal studies conducting using identical methods in 1990/1993 and 2008/2011. The authors assert that all procedures contributing to this work comply with the ethical standards of the relevant national and institutional committees on human experimentation and with the Helsinki Declaration of 1975, as revised in 2008. All procedures involving human subjects were approved by local and multi-centre ethical committees (CFAS I: REC99/5/22, 05/MRE05/37; CFAS II: 07/MRE05/48).

Data were obtained from the first waves of the Cognitive Function and Ageing Studies: CFAS I and CFAS II. The CFAS studies are population-based longitudinal studies of ageing, where participants aged 65 and older were randomly selected from the Family Health Service Authority lists from specific areas of England and Wales, and include community-based participants and those in long-term care.

Potential participants were initially contacted via a letter from their general practice. If the potential participant provided written informed consent, this was followed by a visit from a trained interviewer (often from a healthrelated background) from a team of interviewers. The interview was conducted in the participants' place of residence using a structured, computer assisted interview with direct data entry.

For potential participants considered to lack mental capacity, as per the Mental Capacity Act in the UK, a request to a key informant, usually a close family member, was made for an interview. The interviews were then performed with the assistance of a proxy (often a close family member).

Participants were interviewed between December 1990 and July 1993 (CFAS I), and between November 2008 and October 2011 (CFAS II).

Data from three centres from CFAS I of Cambridgeshire (rural), Newcastle (urban) and Nottingham (urban) were chosen to match the centres in CFAS II. Sampling was stratified by age group (65-74 vs $\geq 75$ years). CFAS I and CFAS II had very similar designs and assessment methods and so medication use prevalence estimates can be directly compared [18]. The response rate was $80 \%$ in CFAS I and 56\% in CFAS II [18]. Inverse probability weights are available for both studies to ensure estimates reflect the age and sex structure of their respective populations. CFAS I and II assessments include questions about socio-demographic characteristics, residence (long-term care or community-dwelling), medications, health, activities of daily living (basic and instrumental), and tests of cognitive function [18]. For data access and study information visit www.cfas.ac.uk. 


\section{Medication exposure}

Medication usage, both prescribed and over the counter, was obtained by self-report. At interview, participants were asked 'Are you currently taking any medicines, tablets or injections of any kind, either you buy yourself or are prescribed by your doctor?' Where possible packaging was checked, with proxies supplying medication information if required. All prescribed and over the counter medications were recorded using NHS Read codes [2]. Read codes are a computerised comprehensive coded thesaurus used within the NHS. Prescription and OTC medication reported was scored 0 to 3 on the Anticholinergic Burden Scale (ACB). Medication with in-vitro activity, but no clinically relevant effect are scored 1 . Medication with clinically relevant effects are scored 2 or 3 if associated with delirium. Other medications are scored 0 . For the full list visit:

https:/www.uea.ac.uk/documents/3306616/10940915/ Anticholinergics/088bb9e6-3ee2-4b75-b8ce-b2d59dc538c2

Details of the scale development are reported elsewhere $[4,5]$. For medications available in the UK, but not rated on the ACB scale, we applied the same approach used and thus scored (i) all thiazide diuretics, loop diuretics and antihistamines as 1, (ii) all tricyclic antidepressants as 3, and (iii) all creams, eye and ear drops as 0 . CFAS did not record how medications were obtained, and some common medications with potential anticholinergic properties such as chlorphenamine, ranitidine, cimetidine, and codeine products could be prescribed or purchased OTC. We defined any anticholinergic use as the use of any medications scoring 1, 2 or 3 on the ACB scale, potent anticholinergic use as any scoring 3 on the ACB scale, and the anticholinergic burden as the sum of ACB scores for all medications taken.

\section{Population subgroups}

We estimated anticholinergic use in groups defined by sex, age (grouped in 5-year age bands), cognitive function (Mini-Mental State Examination [MMSE] $\leq 21$, MMSE 22-25, and MMSE 26-30 points) [19], and disability [measured by impairments in modified Townsend activities of daily living (ADL) and instrumental activities of daily living (IADL)] [20].

\section{Statistical analysis}

We estimated the prevalence of any anticholinergic medication use, potent anticholinergic use and the average anticholinergic burden, using inverse probability weights that accounted for non-response [18]. To compare cohorts, estimates were standardised to the 2011 UK age and sex distribution, using 5-year age bands, to account for changes in population structure. Prevalences were also estimated in the pre-defined population subgroups. Participants with missing disability or MMSE data were excluded from those comparisons.
We used logistic regression to estimate odds ratios (OR) for 'potent' and 'any' anticholinergic use in CFAS II compared to CFAS I. We used negative binomial regression to estimate the rate ratio comparing the anticholinergic burden between the two cohorts, as anticholinergic burden was an over-dispersed discrete variable. The differences between cohorts were adjusted for age, sex and centre and weighted for non-response. We also tested for interaction effects between subgroups and CFAS cohort to identify different trends over time among the different groups of the older population.

Finally, the prevalence of the potent anticholinergic medication by urological, antispasmodic, antipsychotic, antidepressant, anxiolytic, parkinsonian and antihistamine classes was estimated in CFAS I and II.

\section{Results}

Population characteristics

CFAS I and II included data from 7635 and 7762 participants, respectively. Table 1 summarises the characteristics

Table 1 Baseline participant characteristics in CFAS I and CFAS II

\begin{tabular}{|c|c|c|}
\hline Demographic characteristics & CFAS I $(\boldsymbol{N}=7,635)$ & CFAS $\|(\boldsymbol{N}=7,762)$ \\
\hline \multicolumn{3}{|l|}{ Sex } \\
\hline Male & 3045 (39.9) & $3534(45.5)$ \\
\hline Female & $4590(60.1)$ & $4228(54.5)$ \\
\hline \multicolumn{3}{|l|}{ Age } \\
\hline $64-69$ & $1981(25.9)$ & $1939(25.0)$ \\
\hline 70-74 & $1776(23.3)$ & $1873(24.1)$ \\
\hline $75-79$ & $1725(22.6)$ & $1624(20.9)$ \\
\hline $80-84$ & $1308(17.1)$ & $1278(16.5)$ \\
\hline $85-89$ & $615(8.1)$ & $737(9.5)$ \\
\hline $90+$ & $230(3.0)$ & $311(4.0)$ \\
\hline \multicolumn{3}{|l|}{ Centre } \\
\hline Cambridgeshire & $2601(34.1)$ & $2558(33.0)$ \\
\hline Newcastle & $2522(33.0)$ & $2582(33.3)$ \\
\hline Nottingham & $2512(32.9)$ & $2622(33.8)$ \\
\hline \multicolumn{3}{|l|}{ MMSE $^{1}$} \\
\hline Median (IQR) & $27(24,28)$ & $28(26,29)$ \\
\hline \multicolumn{3}{|l|}{ Disability } \\
\hline None & $5236(68.6)$ & $4975(64.1)$ \\
\hline IADL disability & $1048(13.7)$ & $1495(19.3)$ \\
\hline ADL-IADL disability & $1267(16.6)$ & $981(12.6)$ \\
\hline \multicolumn{3}{|l|}{ Residence } \\
\hline Community-dwelling & $7245(94.9)$ & $7565(97.5)$ \\
\hline Long term care & $242(3.2)$ & $197(2.5)$ \\
\hline
\end{tabular}

Cell entries denote $\mathrm{n}(\%)$ unless otherwise specified

1139 and 255 participants had missing MMSE data in CFAS I and CFAS II Abbreviations: CFAS Cognitive Function and Ageing Studies, $\mathrm{Cl}$ confidence interval, MMSE Mini-Mental State Examination, $A D L$ activities of daily living, IADL instrumental activities of daily living, $S D$ standard deviation, $I Q R$ Interquartile range 
of CFAS I and II participants. Although the mean and Standard Deviation (SD) age were similar, 75.3 (7.1) for CFAS I vs 75.7 (7.3) for CFAS II, there was a greater proportion aged over 85 years in CFAS II. Participants of CFAS II were also slightly more likely to be men and have more IADL disability than in CFAS I.

\section{Potent anticholinergic use}

The overall prevalence of potent anticholinergic use among the over $65 \mathrm{~s}$ increased from $5.7 \%$ (95\% CI $5.2-$ 6.3\%) to $9.9 \%$ (95\% CI 9.3-10.7\%) between CFAS I and II. After adjusting for demographic differences, the odds ratio for this increase was 1.90 (95\% CI 1.67-2.16) (Table 2).

In CFAS II, $12.8 \%$ of women used a potent anticholinergic compared to $7.0 \%$ of men. This is approaching twice the rate in CFAS I for both sexes. Potent anticholinergic use was not strongly related to age; but the heaviest users in CFAS II were those with clinically significant cognitive impairment (16.5\% [95\% CI 12.022.3\%] of those with an MMSE of 21 or less) and more disability, with $20.8 \%$ (95\% CI $17.6-24.5 \%$ ) of the most disabled using a potent anticholinergic compared to
6.3\% (95\% CI 5.6-7.1\%) of those with no disability. The greatest rate of increase between cohorts was seen among those with IADL disability (from 6.8\% [95\% CI 5.4-8.7\%] in CFAS I to $15.8 \%$ [95\% CI 13.8-18.0\%] in CFAS II, $p$-value for interaction $=0.05$ ).

The increases in potent anticholinergic use were driven by an increased use of anticholinergic urologicals and antidepressants (Table 3). Use of potent anticholinergic urologicals and antidepressants increased from $0.3 \%$ (95\% CI $0.2-0.4 \%)$ to $2.8 \%$ (95\% CI $2.4-3.2 \%)$ and $4.0 \%$ (95\% CI $3.6-4.5 \%$ ) to $5.9 \%$ (95\% CI $5.4-6.5 \%$ ) between CFAS I and CFAS II, respectively. The most common anticholinergic urologicals used in CFAS II were oxybutynin (35\% of anticholinergic urological drugs), tolterodine (31\%) and solifenacin (17\%), and the most common anticholinergic antidepressant reported in CFAS II was amitriptyline (69\% of anticholinergic antidepressant drugs).

\section{Any anticholinergic use}

The prevalence of medication use with 'any' anticholinergic activity increased from $49.6 \%$ (95\% CI 48.4-50.7\%)

Table 2 Prevalence of any and potent anticholinergic use in CFAS I and CFAS II, by age, sex, cognition and disability

\begin{tabular}{|c|c|c|c|c|c|c|c|c|}
\hline \multirow{3}{*}{ Population } & \multicolumn{4}{|c|}{ Any anticholinergic use } & \multicolumn{4}{|c|}{ Potent anticholinergic use } \\
\hline & \multicolumn{2}{|c|}{ Prevalence \% (95\% Cl) } & \multicolumn{2}{|c|}{$\begin{array}{l}\text { Adjusted OR for } \\
\text { CFAS II vs CFAS |a }\end{array}$} & \multicolumn{2}{|c|}{ Prevalence \% (95\% Cl) } & \multicolumn{2}{|l|}{$\begin{array}{l}\text { Adjusted OR for } \\
\text { CFAS II vs CFAS | }\end{array}$} \\
\hline & CFAS I & CFAS ॥ & OR $(95 \% \mathrm{Cl})$ & $p^{b}$ & CFAS I & CFAS ॥ & OR $(95 \% \mathrm{Cl})$ & $p^{b}$ \\
\hline Overall & $49.6(48.4,50.7)$ & $64.3(63.2,65.4)$ & $1.25(1.17,1.34)$ & $<0.001$ & $5.7(5.2,6.3)$ & $9.9(9.3,10.7)$ & $1.90(1.67,2.16)$ & $<0.001$ \\
\hline \multicolumn{9}{|l|}{ By sex } \\
\hline Male & $46.7(44.9,48.6)$ & $61.3(59.6,62.9)$ & 1.00 & 0.06 & $3.6(3.0,4.4)$ & $6.4(5.6,7.3)$ & 1.00 & 0.59 \\
\hline Female & $51.3(49.8,52.8)$ & $66.7(65.2,68.2)$ & $1.14(0.99,1.30)$ & & $7.0(6.3,7.8)$ & $12.8(11.7,13.9)$ & $1.08(0.82,1.43)$ & \\
\hline \multicolumn{9}{|l|}{ By age, years } \\
\hline $64-69$ & $44.4(42.2,46.6)$ & $53.5(51.2,55.7)$ & 1.00 & $<0.001$ & $5.2(4.3,6.3)$ & $8.0(6.8,9.5)$ & 1.00 & 0.49 \\
\hline $70-74$ & $48.2(45.9,50.6)$ & $62.9(60.7,65.1)$ & $1.33(1.11,1.60)$ & & $5.9(4.9,7.1)$ & $9.9(8.6,11.4)$ & $1.11(0.76,1.60)$ & \\
\hline $75-79$ & $52.8(50.4,55.2)$ & $68.8(66.4,71.0)$ & $1.41(1.17,1.71)$ & & $6.0(4.9,7.2)$ & $11.3(9.7,13.0)$ & $1.28(0.88,1.86)$ & \\
\hline $80-84$ & $54.3(51.5,57.0)$ & $73.0(70.4,75.5)$ & $1.48(1.20,1.82)$ & & $6.8(5.5,8.3)$ & $11.0(9.3,12.9)$ & $1.09(0.73,1.62)$ & \\
\hline $85-89$ & $53.8(49.6,58.0)$ & $72.1(68.5,75.5)$ & $1.58(1.21,2.07)$ & & $5.3(3.7,7.5)$ & $11.8(9.5,14.6)$ & $1.59(0.95,2.69)$ & \\
\hline $90+$ & $50.7(43.5,57.8)$ & $75.5(68.9,81.1)$ & $2.10(1.36,3.22)$ & & $4.1(2.0,8.0)$ & $9.0(5.9,13.6)$ & $1.54(0.63,3.75)$ & \\
\hline \multicolumn{9}{|l|}{ By cognition } \\
\hline MMSE $\leq 21$ & $53.3(49.0,57.6)$ & $71.5(65.0,77.1)$ & 1.00 & 0.02 & $11.2(8.6,14.4)$ & $16.5(12.0,22.3)$ & 1.00 & 0.83 \\
\hline MMSE 22-25 & $52.7(50.2,55.1)$ & $69.9(67.1,72.6)$ & $0.94(0.70,1.25)$ & & $6.7(5.6,8.1)$ & $13.4(11.4,15.6)$ & $1.10(0.71,1.70)$ & \\
\hline MMSE 26-30 & $48.6(47.0,50.2)$ & $62.8(61.5,64.1)$ & $0.76(0.59,0.99)$ & & $4.5(3.9,5.2)$ & $8.4(7.7,9.2)$ & $1.00(0.68,1.49)$ & \\
\hline \multicolumn{9}{|l|}{ By disability } \\
\hline No impairment & $42.4(40.8,44.0)$ & $56.9(55.4,58.5)$ & 1.00 & 0.27 & $3.8(3.3,4.5)$ & $6.3(5.6,7.1)$ & 1.00 & 0.05 \\
\hline IADL impairment & $67.1(64.0,70.0)$ & $80.4(78.1,82.5)$ & $1.13(0.93,1.36)$ & & $6.8(5.4,8.7)$ & $15.8(13.8,18.0)$ & $1.46(1.04,2.04)$ & \\
\hline ADL impairment & $71.1(67.7,74.3)$ & $85.4(82.3,88.1)$ & $1.15(0.92,1.43)$ & & $15.8(13.1,18.9)$ & $20.8(17.6,24.5)$ & $0.95(0.70,1.29)$ & \\
\hline
\end{tabular}

Weighted for nonresponse and standardised by the UK 2011 age population, missing cases excluded

Abbreviations: CFAS Cognitive Function and Ageing Studies, Cl confidence interval, MMSE Mini-Mental State Examination, $A D L$ activities of daily living, IADL instrumental activities of daily living

${ }^{a}$ Adjusted for age, sex and centre

${ }^{\mathrm{b}}$ Global test for the interaction between the covariate and difference in prevalence between CFAS I and CFAS II 
Table 3 Prevalence of potent anticholinergic use in CFAS I and CFAS II, by drug class

\begin{tabular}{lll}
\hline Potent anticholinergic class & 1991 CFAS I & 2011 CFAS II \\
\hline Urological & $0.3(0.2,0.4)$ & $2.8(2.4,3.2)$ \\
Antispasmodic & $0.5(0.3,0.6)$ & $0.3(0.2,0.5)$ \\
Antipsychotic & $1.0(0.8,1.2)$ & $0.9(0.7,1.1)$ \\
Antidepressant & $4.0(3.6,4.5)$ & $5.9(5.4,6.5)$ \\
Anxiolytic & $\mathrm{N} / \mathrm{A}$ & $0.2(0.1,0.3)$ \\
Parkinsonian & $0.2(0.2,0.4)$ & $0.1(0.0,0.2)$ \\
Antihistamine & $0.2(0.1,0.3)$ & $0.5(0.3,0.7)$ \\
\hline
\end{tabular}

Cell entries denote $\%$ prevalence (95\% confidence intervals)

Abbreviations: CFAS Cognitive Function and Ageing Studies

to $64.3 \%$ (95\% CI 63.2-65.4\%) between CFAS I and CFAS II (Table 2, adjusted OR of 1.25; 95\% CI 1.171.34). The greatest increases in use across the 20 years was observed for older participants (from 50.7\% [95\% CI 43.5-57.8\%] in CFAS I to 75.5\% [95\% CI 68.9-81.1\%] in CFAS II for those aged 90 years or more, $p$-value for interaction $<0.001$ ), and in those with clinically significant cognitive impairment (from 53.3\% [95\% CI 49.057.6\%] in CFAS I to $71.5 \%$ [95\% CI 65.0-77.1\%] in CFAS
II for those with an MMSE score of 21 or lower, $p$-value for interaction $=0.02$ ).

\section{Anticholinergic burden}

The average total anticholinergic burden increased from 0.99 (95\% CI 0.96-1.03) in 1991 to 1.11 (95\% CI 1.081.15 ) in 2011, adjusted ratio of 1.12 (95\% CI 1.07-1.17) (Table 4).

Women and older participants had the greatest total burden score, and had experienced the greatest increases since CFAS I. For example the mean ACB score increased from 1.05 (95\% CI 1.01-1.09) in CFAS I to 1.23 (95\% CI 1.18-1.28) in CFAS II for women (p-value for interaction $=0.01)$, and from 0.97 (95\% CI 0.77-1.17) in CFAS I to 1.36 (95\% CI 1.17-1.54) in CFAS II for those aged 90 years or more ( $\mathrm{p}$-value for interaction $<0.001$ ).

\section{Discussion}

The prevalence of potent anticholinergic use in the older population in England nearly doubled between 1990/93 and 2008/11. After adjustment for demographic variables, we found that participants in the later study (CFAS II) were 1.9 times more likely to be on potent anticholinergics as compared to participants in the earlier study (CFAS I).

Table 4 Average anticholinergic burden in CFAS I and CFAS II, by age, sex, cognition and disability

\begin{tabular}{|c|c|c|c|c|}
\hline \multirow[t]{2}{*}{ Population } & \multicolumn{2}{|c|}{ Mean ACB sum $(95 \% \mathrm{Cl})$} & \multicolumn{2}{|c|}{ Adjusted rate ratio for CFAS $\|$ vs CFAS $\left.\right|^{a}$} \\
\hline & $\overline{C F A S I}$ & CFAS ॥ & Rate ratio $(95 \% \mathrm{Cl})$ & $p^{\mathbf{b}}$ \\
\hline Overall & $0.99(0.96,1.03)$ & $1.11(1.08,1.15)$ & $1.12(1.07,1.17)$ & $<0.001$ \\
\hline \multicolumn{5}{|l|}{ By sex } \\
\hline Male & $0.91(0.86,0.95)$ & $0.96(0.92-1.01)$ & 1.00 & \multirow[t]{2}{*}{0.01} \\
\hline Female & $1.05(1.01,1.09)$ & $1.23(1.18-1.28)$ & $1.12(1.03,1.23)$ & \\
\hline \multicolumn{5}{|l|}{ By age, years } \\
\hline $64-69$ & $0.87(0.81,0.92)$ & $0.81(0.75-0.87)$ & 1.00 & \multirow[t]{6}{*}{$<0.001$} \\
\hline $70-74$ & $0.97(0.91,1.04)$ & $1.10(1.03-1.66)$ & $1.21(1.05,1.38)$ & \\
\hline $75-79$ & $1.09(1.02,1.15)$ & $1.26(1.18-1.34)$ & $1.24(1.09,1.42)$ & \\
\hline $80-84$ & $1.12(1.04,1.20)$ & $1.28(1.19-1.36)$ & $1.22(1.06,1.41)$ & \\
\hline $85-89$ & $1.07(0.95,1.18)$ & $1.37(1.25-1.49)$ & $1.39(1.17,1.66)$ & \\
\hline $90+$ & $0.97(0.77,1.17)$ & $1.36(1.17-1.54)$ & $1.53(1.17,1.99)$ & \\
\hline \multicolumn{5}{|l|}{ By cognition } \\
\hline MMSE $\leq 21$ & $1.29(1.13,1.46)$ & $1.32(1.13,1.52)$ & 1.00 & \multirow[t]{3}{*}{0.03} \\
\hline MMSE 22-25 & $1.09(1.02,1.16)$ & $1.38(1.28,1.48)$ & $1.11(0.94,1.31)$ & \\
\hline MMSE 26-30 & $0.93(0.89,0.97)$ & $1.04(1.01,1.09)$ & $0.97(0.83,1.13)$ & \\
\hline \multicolumn{5}{|l|}{ By disability } \\
\hline No impairment & $0.76(0.72,0.79)$ & $0.85(0.81,0.89)$ & 1.00 & \multirow[t]{3}{*}{0.91} \\
\hline IADL impairment & $1.51(1.40,1.61)$ & $1.64(1.54,1.74)$ & $1.02(0.92,1.13)$ & \\
\hline ADL impairment & $1.85(1.71,1.99)$ & $1.88(1.74,2.03)$ & $1.02(0.91,1.14)$ & \\
\hline
\end{tabular}

Prevalence (95\% confidence interval) displayed, weighted for nonresponse and standardised by the UK 2011 age population, with missing cases excluded Abbreviations: $A C B$ Anticholinergic Cognitive Burden scale, CFAS Cognitive Function and Ageing Studies, MMSE Mini-Mental State Examination, ADL activities of daily living, $I A D L$ instrumental activities of daily living

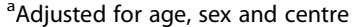

${ }^{\mathrm{b}}$ Global test for the interaction between the covariate and ratio of total anticholinergic burden between CFAS I and CFAS II 
This was mainly due to increases in the availability and use of anticholinergic urologicals (common drugs were oxybutynin, solifenacin and tolterodine) and antidepressants (the most common being amitriptyline). More than one in five of those with an impairment in activities of daily living, and one in six of those with MMSE less than 21 , indicating clinically significant cognitive impairment including dementia, reported use of a potent anticholinergic medication in CFAS II, both significantly higher than in CFAS I [21]. This is despite guidance suggesting cautious use of these drugs. Those with IADL disability had the greatest disproportionate increases in potent anticholinergic use. Women and older participants also had disproportionately greater increases in total anticholinergic burden between study periods.

A number of studies have described changes in the rates of anticholinergic prescribing [16, 17]. A study in Scotland examined changes in the numbers of prescriptions of anticholinergic medications, from 1995 to 2010, and found a statistically significant but modest increase in the number of older people prescribed any anticholinergic (20.7\% vs $23.7 \%, p<0.001)[16]$. A repeated cross-sectional analysis of office-based outpatient visits for older people in the USA found that the prevalence of high-risk anticholinergic prescriptions was stable from 2006 to 2015; it increased from $6.1 \%$ in $2006-07$ to $6.8 \%$ in $2008-09$ and decreased to $4.7 \%$ by $2014-15$ [17]. However, previous studies have not been able to include over-the-counter medication use nor describe use in vulnerable patient groups; the US study also only included prescriptions issued by the physician at the sampled visit.

We observed an increase in anticholinergic urological use between 1991 and 2011, partly because many of the commonly used urologicals were only introduced in the 1990s, or later. Other studies have also reported increases in the prescribing of anticholinergic urologicals [16, 22, 23]. A $23 \%$ increased number of new users of anticholinergics for overactive bladder was reported in a UK study (from 12, 598 in 2004 to 15,441 in 2012) [23]. A significant increase in the proportion of women presenting to physicians with urinary incontinence then prescribed bladder anticholinergics was also reported in the US $(16.7 \%$ in 1999 to $35.0 \%$ in 2009, $p=0.006$ ) [22].

Use of anticholinergic antidepressants also increased between CFAS I and II; confirming other studies [24]. In addition to anticholinergic effects, antidepressants are associated with hyponatraemia $[25,26]$. Even mild hyponatraemia induced by antidepressants may worsen cognition and cause falls compounding apparent anticholinergic effects [25]. Depression is also an early sign of dementia and therefore older people with depression may be particularly vulnerable to cognitive anticholinergic effects [27].

Anticholinergics can have a significant impact on morbidity in older people particularly those living with any form of clinically significant cognitive impairment including dementia [7, 12]. Anticholinergics can worsen dementia, cause numerous anticholinergic effects, both centrally and peripherally, and may be associated with an excess mortality [7, 12]. Equally importantly, anticholinergics could also worsen the quality of life of the older person and any informal (family) carer [28].

The large increase in the use of potent anticholinergics among people with clinically significant cognitive impairment and physical disabilities is particularly concerning. There is increasing evidence, from recent research, that such usage is associated with an increased risk of dementia $[10,11,12]$. Furthermore, anticholinergic cognitive effects are likely to have more severe consequences, such as medication errors, in people with less cognitive reserve for example dementia or traumatic brain injury [29]. Medication management itself is an instrumental activity of daily living with high demands on memory and executive function [30], and so anticholinergic induced cognitive impairment may increase the risk of both non-adherence to medication, and medication errors [30, 29]. This in turn will increase dependency on informal carers worsening the burden on informal carers [31].

\section{Strengths and weaknesses}

Frail older people with multi-morbidities including those with dementia are frequently excluded from controlled trials, and so effectiveness of anticholinergics is rarely directly assessed, and observational studies are vital for monitoring risks. Strengths of this study include the population-based sampling in CFAS from the same geographic areas 20-years apart and to ascertain key patient characteristics, cognition and disability associated with medication use. Although most anticholinergics are prescribed, a further strength of our study was the ability to more accurately capture the full range of anticholinergic use, by including OTC medications.

This appropriateness of prescribing was not assessed as part of the CFAS study. The increase in use of anticholinergics might reflect improvements in diagnosis and better access to treatment for conditions such as incontinence, depression and pain. Such conditions can be very debilitating, and for clinicians and patients the key issue is balancing the risks versus the benefits.

Our study has some limitations. The accuracy of the self-reported medication use and the duration of treatment is unknown. Although, to increase the accuracy of the reporting, interviewers requested, where possible, to see the medication packages (and repeat prescription scripts) to enter correct drug names, we cannot be sure if the participants were adherent to the medication. The data used is from 1990 to 1993 and 2008 to 2011 and therefore we recommend that the study is repeated with more recent data to examine whether the trends continue. 
Studies examining UK trends in anticholinergic medication use post 2011 are rare. Increased prescribing of anticholinergics for overactive bladder has been reported until 2012 for adults [23]. Warnings against antipsychotic use in dementia has decreased prescribing to these patients [32], but we lack information on the general older population. Antidepressant prescribing has been increasing from 2013 to 18, but detail has not been provided by anticholinergic antidepressants or for older people specifically [33].

We used the ACB scale to identify anticholinergic medications, however this is one of 18 different scales that all vary in their content and how they are derived and how anticholinergic activity is quantified [34]. However, the scales closely agree on which medications they classify as potently anticholinergic. The response rate was lower in CFAS II, and it is not clear whether this would underestimate or over-estimate medication use in this cohort. We used inverse probability weights to correct age and sex distributions for non-response, and conducted analyses stratified by levels of cognitive function and disability, and so our findings are unlikely to be biased by differential non-response between cohorts [18]. Our study is descriptive and we did not have sufficient comorbidity data to sufficiently examine why older people in the various subgroups had increased anticholinergic use, but increased diagnoses of conditions for which anticholinergics are indicated for is likely a factor.

\section{Future research}

Further research is needed to monitor anticholinergic use within vulnerable populations, particularly older people living with clinically significant cognitive impairment including dementia, in the UK since 2011 and in other countries. We also need a clearer understanding of the relative risk versus benefit of anticholinergics and in whom the risk is greatest, and the effectiveness of interventions to reduce the harm associated with anticholinergics. Interventions to limit the use of inappropriate anticholinergics require development and testing; a realist approach, which focuses on the key importance of context and mechanism offers a promising avenue for such intervention development [35-37].

\section{Conclusions}

In summary the use of potent anticholinergics nearly doubled in the older population in England over an appropriate 20 year period (from 1990/93 to 2008/11), largely due to rising use of antidepressants and urologicals. The use of anticholinergics is highest among the most vulnerable groups including people living with clinically significant cognitive impairment. This raises concerns as anticholinergic medications are associated with a range of side-effects including cognitive decline.

\section{Abbreviations}

ACB: Anticholinergic Cognitive Burden; ADL: Activities of daily living; CFAS: Cognitive Function and Ageing Studies; Cl: Confidence Interval; IADL: Instrumental activities of daily living; MMSE: Mini Mental State Examination; NHS: National Health Service; OR: Odds ratios; OTC: Over the counter; SD: Standard Deviation

\section{Acknowledgements}

We would like to thank the UK Medical Research council CFAS study group for data collection and management. We are also grateful to all respondents, their families and their primary care teams for their participation in the CFAS studies. We thank the CFAS II fieldwork interviewers at Cambridge, Nottingham and Newcastle for their valuable contribution.

\section{Authors' contributions}

$\mathrm{KR}, \mathrm{GS}, \mathrm{CG}$ and IM formulated the research question. CG coded the data, and $K R$ and $C G$ conducted the analysis with GS and FEM providing input on the analysis. IM advised on medication aspects. CF, AA, YL, NS, CB, LR and PKM provided expert clinical input. KR, CG and IM drafted and wrote the article with support from all co-authors. All authors approved the final draft.

\section{Funding}

This research was supported by funding from Alzheimer's Society (AS-PG2013-017). The funder had no role in the design, implementation, analysis or interpretation of the study.

\section{Availability of data and materials}

Data can be shared through application. For further information please refer to the application forms on the website http://www.cfas.ac.uk/cfas-i/data/ \#cfasi-data-request

\section{Ethics approval and consent to participate}

Written consent was obtained for participation in the CFAS study. Ethical approval was obtained locally at all sites from 1991 and at Multi-centre research ethics committees (CFAS I: REC99/5/22, 05/MRE05/37; CFAS II: 07) MRE05/48) during the course of the study. For example, the first multi-centre ethical approval was obtained at the Anglia and Oxford multi-centre research ethics committee - part of the NHS REC (ref: 99/5/22). We requested the data from the CFAS National Coordinator. Data applications were reviewed by the Co-operative Management Committee (CMC) of CFAS. Datasets were created and released by CFAS Data Managers.

Further detail on all ethical approvals can be found at: http://www.cfas.ac.uk/ files/2015/07/Ethical-approvals-for-CFAS.pdf.

Written informed consent was obtained from every participant. If the potential participant was considered to lack mental capacity, as per the Mental Capacity Act in the UK, assent was obtained from a key informant, usually a close family member. This method of consent was approved by an ethics committee.

\section{Consent for publication}

Not applicable.

\section{Competing interests}

Prof. Fox and Dr. Maidment received travel grants and Profs Fox and Myint received lecture fees from Astellas Pharma UK. Prof. Loke received consultancy fees from Thame Pharmaceuticals. All other authors report no conflict of interest.

\section{Author details}

${ }^{1}$ University of East Anglia, Norwich, UK. ${ }^{2}$ University of East Anglia, Quadram Institute Bioscience, Norwich Research Park, Norwich, UK. ${ }^{3}$ University of Cambridge, Cambridge, UK. ${ }^{4}$ University of Newcastle, Newcastle, UK.

${ }^{5}$ University of Aberdeen, Aberdeen, UK. ${ }^{6}$ Aston University, Birmingham B4 7ET, UK

Received: 18 November 2019 Accepted: 16 July 2020 Published online: 31 July 2020

\section{References}

1. Office for National Statistics. Overview of the UK population: November 2018. 2018 
2. Gao L, Maidment I, Matthews FE, Robinson L, Brayne C. Medication usage change in older people (65+) in England over 20 years: findings from CFAS I and CFAS II. Age Ageing. 2018;47:220-5.

3. Arthur A, Savva G, Barnes L, et al. Changing prevalence and treatment of depression among the over-65s over two decades: findings from the cognitive function and ageing studies. Br J Psychiatry. 2019;216:1-6.

4. Campbell N, Boustani M, Limbil T, et al. The cognitive impact of anticholinergics: a clinical review. Clin Interv Aging. 2009;4:225-33.

5. Boustani M, Campbell N, Munger S, Maidment I, Fox C. Impact of anticholinergics on the aging brain: a review and practical application. Aging Health. 2008;4.

6. Puhan MA, Bachmann LM, Kleijnen J, ter Riet G, Kessels AG. Inhaled drugs to reduce exacerbations in patients with chronic obstructive pulmonary disease: a network meta-analysis. BMC Med. 2009;7:1-14.

7. Fox C, Richardson K, Maidment ID, et al. Anticholinergic medication use and cognitive impairment in the older population: the Medical Research Council cognitive function and ageing study. J Am Geriatr Soc. 2011;59:1477-83.

8. Richardson K, Bennett K, Maidment ID, Fox C, Smithard D, Kenny RA. Use of medications with anticholinergic activity and self-reported injurious falls in older community-dwelling adults. J Am Geriatr Soc. 2015;63:1561-9.

9. Kim SJ, Jung D, Shim JC, et al. The effect of anticholinergic burden on cognitive and daily living functions in patients with schizophrenia. Asian J Psychiatr. 2019:46:111-7.

10. Coupland CAC, Hill T, Dening T, Morriss R, Moore M, Hippisley-Cox J. Anticholinergic drug exposure and the risk of dementia. JAMA Intern Med. 2019;179:1-10.

11. Gray SL, Anderson ML, Dublin S, et al. Cumulative use of strong Anticholinergics and incident dementia. JAMA Intern Med. 2015;175:401.

12. Richardson K, Fox C, Maidment I, et al. Anticholinergic drugs and risk of dementia : case-control study. BMJ. 2018:1-12.

13. Gray SL, Hanlon JT. Anticholinergic medication use and dementia: latest evidence and clinical implications. Ther Adv Drug Saf 2016; 2042098616658399.

14. Fox C, Smith T, Maidment I, et al. Effect of medications with anti-cholinergic properties on cognitive function, delirium, physical function and mortality: a systematic review. Age Ageing. 2014;43:604-15.

15. Kachru N, Carnahan RM, Johnson ML, Aparasu RR. Potentially inappropriate anticholinergic medication use in community-dwelling older adults: a National Cross-Sectional Study. Drugs Aging. 2015;32:379-89.

16. Sumukadas D, McMurdo MET, Mangoni AA, Guthrie B. Temporal trends in anticholinergic medication prescription in older people: repeated crosssectional analysis of population prescribing data. Age Ageing. 2014;43:515-21.

17. Rhee TG, Choi YC, Ouellet GM, Ross JS. National prescribing trends for high-risk anticholinergic medications in older adults. J Am Geriatr Soc. 2018;66:1382-7.

18. Matthews FE, Arthur A, Barnes LE, et al. A two-decade comparison of prevalence of dementia in individuals aged 65 years and older from three geographical areas of England: results of the cognitive function and ageing study i and II. Lancet. 2013;382:1405-12.

19. Folstein MF, Folstein SE, McHugh PR. 'Mini-mental state'. A practical method for grading the cognitive state of patients for the clinician. J Psychiatr Res. 1975;12:189-98.

20. MRC CFAS Study. Cognitive function and dementia in six areas of England and Wales: the distribution of MMSE and prevalence of GMS organicity level in the MRC CFA study. Psychol Med. 1998;28:319-35.

21. National Institute for Health and Care Excellence. Dementia: assessment, management and support for people living with dementia and their carers. London: NICE; 2018. p. 540-0. https://www.nice.org.uk/guidance/ng97/ resources/dementia-assessment-management-and-support-for-people-livingwith-dementia-and-their-carers-pdf-1837760199109 (Accessed 25 June 2019).

22. Forde JC, Chughtai B, Cea M, Stone BV, Te A, Bishop TF. Trends in ambulatory management of urinary incontinence in women in the United States. Female Pelvic Med Reconstr Surg. 2017;23:250-5.

23. Margulis AV, Linder $M$, Arana $A$, et al. Patterns of use of antimuscarinic drugs to treat overactive bladder in Denmark, Sweden, and the United Kingdom. PLoS One. 2018;13:1-13.

24. Lockhart $P$, Guthrie B. Trends in primary care antidepressant prescribing 1995-2007. Br J Gen Pr. 2011;61:565-72.

25. Leth-Møller KB, Hansen AH, Torstensson M, et al. Antidepressants and the risk of hyponatremia : a Danish register-based population study. BMJ Open. 2016;6:1-8.
26. Daniel S, Amir S, Bronislava C, Diker-Cohen T, Rozen-Zvi B, Gafter-Gvili A. Medication induced SIADH: distribution and characterization according to medication class. Br J Clin Pharmacol. 2017;83(8):1801-7.

27. Goodarzi ZS, Mele BS, Roberts DJ, Holroyd-Leduc J. Depression case finding in individuals with dementia: a systematic review and meta-analysis. J Am Geriatr Soc. 2017;65(5):937-48.

28. Aston L, Hilton A, Moutela T, Shaw R, Maidment I. Exploring the evidence base for how people with dementia and their informal carers manage their medication in the community: a mixed studies review. BMC Geriatr. 2017;17:242.

29. Maidment ID, Haw C, Stubbs J, Fox C, Katona C, Franklin BD. Medication errors in older people with mental health problems: a review. Int J Geriatr Psychiatry. 2008;23:564-73.

30. Foster ER. Instrumental activities of daily living performance among people with parkinson's disease without dementia. Am J Occup Ther. 2014;68:353-62

31. Maidment ID, Aston L, Moutela T, Fox CG, Hilton A. A qualitative study exploring medication management in people with dementia living in the community and the potential role of the community pharmacist. Health Expect. 2017;20:929-40.

32. Martinez C, Jones RW, Rietbrock S. Trends in the prevalence of antipsychotic drug use among patients with Alzheimer's disease and other dementias including those treated with antidementia drugs in the community in the UK: a cohort study. BMJ Open. 2013;3:e002080.

33. Balinskaite $V$. Trends and geographical variation in antidepressant prescribing in primary care in England: a descriptive analysis. Lancet. 2019; 394:S21

34. Welsh TJ, van der Wardt V, Ojo G, Gordon AL, Gladman JRF. Anticholinergic drug burden tools/scales and adverse outcomes in different clinical settings: a systematic review of reviews. Drugs Aging. 2018;35:523-38.

35. Maidment I, Booth A, Mullan J, McKeown J, Bailey S, Wong G. Developing a framework for a novel multi-disciplinary, multi-agency intervention(s), to improve medication management in community-dwelling older people on complex medication regimens (MEMORABLE)--a realist synthesis. Syst Rev. 2017;6:125.

36. Maidment I, Lawson S, Wong G, et al. Towards an understanding of the burdens of medication management affecting older people: the MEMORABLE realist synthesis. BMC Geriatr. 2020;20:183.

37. Maidment ID, Lawson S, Wong G et al. Medication management in older people: the MEMORABLE realist synthesis. Health Serv Deliv Res. 2020;8:26. https://www.journalslibrary.nihr.ac.uk/hsdr/hsdr08260/\#/abstract (Accessed 13 July 2020)

\section{Publisher's Note}

Springer Nature remains neutral with regard to jurisdictional claims in published maps and institutional affiliations.

\section{Ready to submit your research? Choose BMC and benefit from:}

- fast, convenient online submission

- thorough peer review by experienced researchers in your field

- rapid publication on acceptance

- support for research data, including large and complex data types

- gold Open Access which fosters wider collaboration and increased citations

- maximum visibility for your research: over $100 \mathrm{M}$ website views per year

At $\mathrm{BMC}$, research is always in progress.

Learn more biomedcentral.com/submission 